\title{
Recubrimientos de TiN depositados mediante ACPVD sobre aleaciones de magnesio AM60
}

\author{
M. PICHEL ${ }^{1}$, N. CANDELA ${ }^{1}$, R. BAREA ${ }^{1}$, G. CONEJERO ${ }^{1}$, M. CARSí ${ }^{2}$ \\ 'Universidad Nebrija, Escuela Politécnica Superior y de Ingeniería. C/ Pirineos 55, 28040 Madrid. Tlf: +34 914521100. \\ e-mail: mpichel@nebrija.es \\ ${ }^{2}$ CENIM. Avda. Gregorio del Amo 8, 28040 Madrid
}

\begin{abstract}
Las aleaciones de magnesio están alcanzando especial interés gracias a sus buenas propiedades específicas, bajo coste y buenas propiedades de moldeabilidad. No obstante su baja dureza, resistencia a desgaste y corrosión, limita sus aplicaciones en ciertos campos de la industria. Estos inconvenientes se pueden solucionar aplicando recubrimientos duros cerámicos, como nitruros o carburos metálicos. El TiN es uno de los más utilizados debido a su alta adherencia, dureza, bajo coeficiente de fricción y estabilidad química.

La deposición física en fase vapor mediante arco catódico, ACPVD, es una técnica muy versátil, que emplea bajas temperaturas y altas energías de ionización, generando recubrimientos de bajo espesor, homogéneos y de calidad. Para alcanzar una alta calidad en los recubrimientos es necesario un exhaustivo control de los parámetros de fabricación, como son la tensión de polarización, el flujo de gas, la intensidad, etc.

El presente artículo estudia la aleación de magnesio, AM60, recubierta con TiN mediante, ACPVD a diferentes valores de intensidad (40A y 100A) y diferente preparación superficial (desbaste hasta grano 4000 y pulido hasta $3 \mu \mathrm{m}$ ). Se incluye una condición final donde se introduce una capa de Al intermedia. Las muestras se caracterizaron mediante difracción de rayos X, rugosidad, microscopía óptica y electrónica de barrido.
\end{abstract}

Palabras clave: Recubrimientos, Métodos de deposición, TiN, ACPVD, Microestructura.

\section{Deposition of TiN coatings using ACPVD technique on AM60 alloy}

Magnesium alloys are reaching special interest due to their good specific properties, low cost and good manufacturing properties. However, their low hardness, wear and corrosion resistance limit their applications in certain sectors of industry. These drawbacks can be solved by applying hard ceramic coatings, such as nitrides or metal carbides. TiN is one of the most used coatings due to its high adhesion, hardness, low coefficient of friction and chemical stability.

Physical vapor deposition by cathodic arc CAPVD, is a versatile technique, which uses low temperatures and high ionization energies, generating homogeneous coatings. To achieve coatings with high quality, a careful control of the manufacturing parameters is required, such as bias voltage, gas flow or intensity.

This paper focuses on magnesium alloys, AM60, coated with TiN using physical vapor deposition cathodic arc technique (CAPVD) at different intensity values (40A and 100A) and surface preparation (grinding up to 4000 grit and polished to $3 \mu \mathrm{m})$. It was included a final condition with an intermediate Al film. The samples were characterized by X-ray diffraction, roughness, optical microscopy and scanning electron.

Keywords: Coatings, Methods of Deposition, TiN, CAPVD, Microstructure.

\section{INTRODUCCIÓN}

Elmagnesio es un metal que ofrece muy buenas propiedades específicas gracias a su baja densidad, lo que le sitúa entre en uno de los materiales más atractivos para el sector de la automoción y aeronáutica [1,2]. No obstante, su baja dureza y baja resistencia a la corrosión y al desgaste presenta un gran inconveniente para ciertos sectores industriales [3]. Estas limitaciones pueden ser mejoradas mediante la deposición de capas delgadas cerámicas en su superficie. Entre los recubrimientos duros cerámicos, el TiN es uno de los más utilizados debido a su alta dureza, resistencia y estabilidad química [4]; estas propiedades están influidas por el tipo de crecimiento que presente el recubrimiento policristalino, que a su vez dependerá de las propiedades elásticas y difusivas del material [5].

La deposición física en fase vapor mediante arco catódico (ACPVD), es una técnica ampliamente usada en la fabricación de recubrimientos delgados duros que presenta una alta versatilidad en la producción de capas metálicas o cerámicas a bajas temperaturas, generando recubrimientos homogéneos y de alta calidad [6]. La evaporación del material se realiza mediante descargas sobre el cátodo, a altas corriente y bajos voltajes. La gran ventaja que presenta, es su alto grado de 
ionización en las especies evaporadas, que promueve la deposición de capas densas a bajas temperatura, gracias a la alta movilidad adatómica que se genera. Dentro de las aplicaciones industriales, las temperaturas del substratro (Ts) son aproximadamente de 0,2-0,3 la temperatura de fusión (Tm) ocurriendo el proceso en condiciones de no equilibrio. Este hecho limita cinéticamente el crecimiento, afectando por ende a la adherencia capa-substrato [7].

El magnesio posee una temperatura de fusión de $660{ }^{\circ} \mathrm{C}$, restringiendo su procesado a trabajar a bajas temperaturas y con un exhaustivo control de sus parámetros, a fin de asegurar el buen acondicionamiento del substrato. El uso de estas condiciones tan limitantes afecta directamente a la cinética y crecimiento de la capa [1, 2].

La consecución de un recubrimiento efectivo requiere de la existencia de una buena adherencia. Ésta está condicionada principalmente por el proceso de fabricación utilizado, por los parámetros de deposición y la preparación superficial previa del material base [5,8]. En la técnica ACPVD, los parámetros más influyentes en la calidad del recubrimiento son el voltaje de polarización, la presión de nitrógeno, la corriente del arco, la distancia y la temperatura [9]. Por otro lado, dependiendo del estado superficial del substrato, la adherencia de la capa y el tipo de crecimiento puede ser variado.

En algunos casos, la incorporación de capas intermedias de anclaje mejora la adherencia entre el recubrimiento y el substrato, ya que pueden reducir la diferencia entre los coeficientes de expansión térmica existentes, así como, mejorar la compatibilidad entre los materiales, derivadas de las diferentes redes cristalinas.

Las etapas del proceso del crecimiento de los recubrimientos de TiN sucede en los siguientes pasos: nucleación, crecimiento, coalescencia e interferencia de islas, que producen una estructura continua, y el crecimiento consecuente del espesor. La selección del plano de crecimiento del recubrimiento puede ocurrir en la etapa de nucleación, resultando en un crecimiento epitaxial, o durante la coalescencia o crecimiento del espesor, caso que suele producirse cuando se utilizan materiales policristalinos orientados aleatoriamente [10]. La evolución de este crecimiento de la capa está controlado por la superficie, la difusión, impurezas o el bombardeo energético [11]. Una vez obtenido el recubrimiento, las diferentes microestructuras que pueden obtenerse se reflejan en el modelo estructural de zonas,

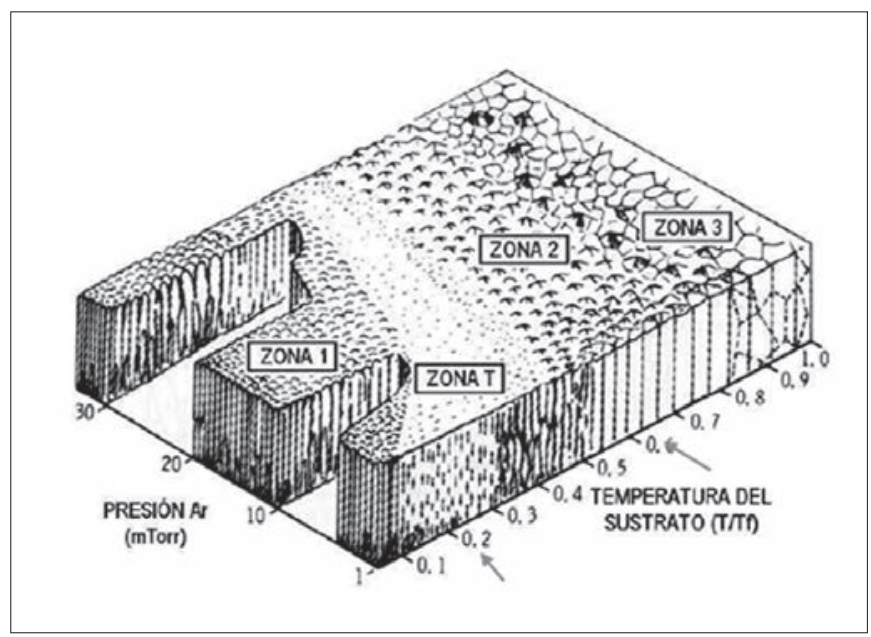

Figura 1: Esquema del modelo de zonas presentado por Thomson [12].
("Structural Zone Model”, SZM) diseñado inicialmente por Thomson [12,13], donde se establecen los diferentes modelos de crecimiento dependientes de la temperatura adquirida en el proceso. En la Figura 1 se muestra un esquema de los diferentes crecimientos que podemos obtener en función de la presión y temperatura.

El modelo de zonas de Thomson se amplió posteriormente teniendo en cuenta otros factores como la energía de crecimiento de los iones, el espesor del film, la energía de los iones bombardeantes o el flujo de gas [14,15]. Mathieu [16] dividió la zona I de crecimiento en tres, la Figura 2(a-e) muestra los diversos tipos de crecimiento planteados. Cuando la energía de los iones hacia el substrato es muy baja o casi inapreciable, los átomos que llegan no poseen casi movilidad por lo que se adhieren a la superfie en el mismo lugar en el que impactaron inicialmente, son las zonas Ia (Figura 2a) y $\mathrm{Ib}$ (Figura 2b). En este caso, el recubrimiento es amorfo y poroso, formado por granos principalmente equiaxiales. La diferencia entre la zona Ia y Ib es el aumento en la energía de las partículas y por tanto, su energía cinética hacia el film, que hace que el recubrimiento de la zona $\mathrm{Ib}$ sea algo menos poroso por la compresión que ejercen los iones que llegan al film creciente sobre los que están ya depositados, ocluyendo los huecos formados. Es importante saber que no se puede pasar de una zona a otra por incremento de la temperatura, porque aumentando la movilidad atómica no se ejerce ningún efecto en la compresión de los huecos, solamente se realiza mediante el aumento de la energía cinética de los iones. El aumento de la temperatura produce un cambio a la zona Ic (Figura 2c), ya que favorece la movilidad y las partículas llegadas al substrato son capaces de difundir en el propio grano y empezar a nuclear en forma de islas, facetadas por el crecimiento más lento de los granos vecinos. En esta zona de crecimiento no se desarrollan orientaciones cristalográficas claras. El recubrimiento final consistirá en columnas rectas facetadas, con menor porosidad por cierre de los huecos en el crecimiento es su superficie pero teniendo una orientación aleatoria porque la nucleación ocurrió de forma aleatoria.

Cuando aumentamos el flujo energético hacia el substrato o la temperatura es mayor a 0,3Tm (Temperatura de fusión), las partículas adquieren mayor energía y movilidad pudiendo difundir de un grano a otro, lo que permite una competición entre los granos orientados en las direcciones más rápidas de crecimiento, es la conocida zona T (Figura 2d); en el caso del TiN, la dirección del plano $\left(\begin{array}{lll}1 & 1 & 1\end{array}\right)$ es la que posee mayor tasa de crecimiento perpendicular presenta, mientras que el plano (2 00 ) es el de mayor tasa de crecimiento paralelo a la dirección del recubrimiento. El film en esta etapa se caracterizará por columnas en forma de $\mathrm{V}$, facetadas y con una dirección preferente de crecimiento.

Finalmente, en el caso que la energía de las partículas sea muy alta puede darse el fenómeno de recristalización, aspirando el film a adquirir la mínima energía superficial, zona II (Figura 2e), es decir, el plano (2 00 ). Los granos orientados en este plano consumirán a los otros dando como resultado un crecimiento de columnas orienadas.

No obstante, en los diferentes tipos de crecimiento la temperatura no es el único factor determinante, sino que aparecen otros, como por ejemplo, el flujo de gas, el tipo de iones incidentes o el espesor del recubrimiento. Por ejemplo, el espesor a su vez, puede depender de otros factores, como son la intensidad y el voltaje de polarización. 

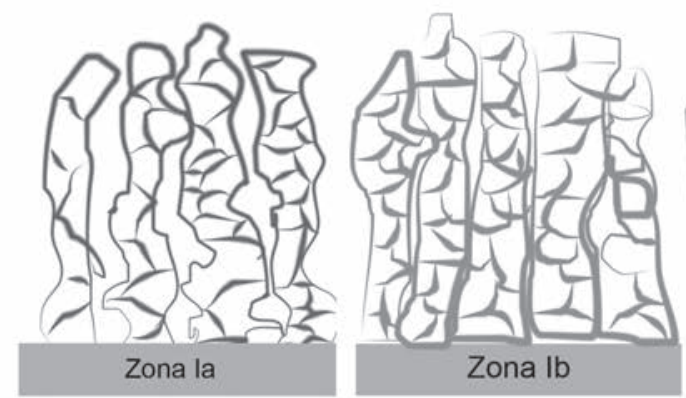
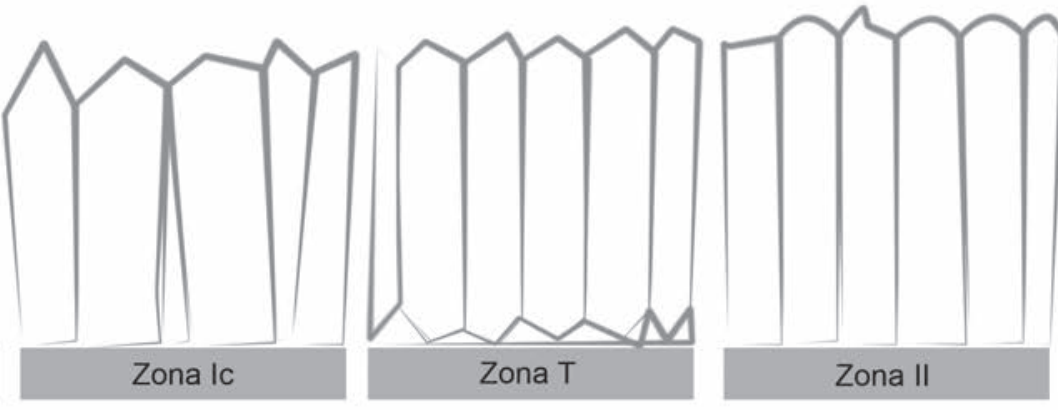

Figura 2: Esquema de las microestructuras del modelo estructural de zonas "SZM".

La microestructura de TiN, para la mayoría de las condiciones, se caracteriza por estar compuesta por granos columnares alargados a lo largo de la dirección de crecimiento, estando algunos de estos cristales extendidos a lo largo de todo el recubrimiento (los recubrimientos fabricados por ACPVD a menudo tiene pocas micras de espesor) [17]. Estos granos poseen fuertes propiedades anisótropas, condicionando su propio crecimiento. No obstante, dependiendo de los parámetros de procesado, también pueden aparecer granos equiaxiales o combinación de equiaxiales y columnares, con variaciones del grado de porosidad en el recubrimiento o la interfase. Zhang y Zhu [18] muestran en sus investigaciones que recubrimientos fabricados sin la aplicación de un voltaje de polarización presentan una estructura columnar abierta, de baja dureza y pobre resistencia al desgaste, mientras que los recubrimientos sobre los que sí se les aplicó un voltaje de polarización son más densos y poseen mayor dureza y resistencia al desgaste.

Estas microestructuras en los recubrimientos de TiN ofrecen a menudo texturas en los planos (2 00 ), (1 111$)$ y (2 20$)$. El plano (111) es aquel que posee mayor coeficiente de difusión perpendicular al substrato, favoreciendo el crecimiento en esta dirección por encima de otros planos, como el $\left(\begin{array}{lll}2 & 0 & 0\end{array}\right)$. Finalmente, el plano (2 20 ) es el plano de menor energía de parada, es aquel que requiere la menor energía de deposición de iones por unidad de superficie a lo largo de una dirección y toma importancia cuando las tensiones son muy elevadas y no pueden acomodarse en el plano de menor energía de deformación $[19,20]$.

No es fácil explicar cuál es la influencia directa entre el crecimiento preferente de estos planos y los parámetros de fabricación, debido a la gran cantidad de técnicas de PVD existentes, que conllevan diferentes parámetros de procesado, así como por la complejidad del mismo proceso de crecimiento bajo atmósferas reactivas [7].

Algunos autores como Mubarak [21] o Nakamura [22], evalúan la relación entre estos parámetros de fabricación con el crecimiento, calidad y propiedades de los recubrimientos, usando substratos de aceros o cerámicos, es decir, materiales con altas temperaturas de fusión. El uso de aleaciones ligeras como el magnesio limita los valores de los parámetros de deposición.

El objetivo principal de este artículo es determinar la influencia de la intensidad de arco, la preparación superficial y la introducción de una capa intermedia de aluminio en la calidad de recubrimientos de TiN fabricados mediante
ACPVD sobre la aleaciones de magnesio AM60 y AZ91; para ello, se estudiará la rugosidad final de las muestras, se realizará una caracterización microestructural mediante microscopía óptica y electrónica de barrido y se evaluarán las fases cristalinas que están presentes en el recubrimiento así como el plano principal de crecimiento de los recubrimientos mediante técnicas de difracción de rayos $\mathrm{X}$.

\section{METODOLOGÍA EXPERIMENTAL}

Se emplearon probetas de $25 \times 25 \times 5 \mathrm{~mm}$ de la aleación de magnesio AM60, aluminio $6 \%$, manganeso 0.26-0.5 \% (ASTM B93 / B93M-09), fabricadas mediante moldeo por inyección.

Las muestras se desbastaron con papel de lija en un ciclo de granulometría 600, 2500 y 4000 y posteriormente se pulieron hasta $3 \mu \mathrm{m}$ con suspensión de diamante hasta obtener una superficie especular. Otro grupo de muestras se dejaron simplemente desbastadas, sin pasar por el ciclo de pulido. Finalmente, fueron limpiadas con un disolvente orgánico. Previamente a la deposición de la capa, se realizó una limpieza mediante un pre-sputtering en la cámara de deposición, bombardeando el substrato con argón durante 3 minutos. Tras la limpieza se procedió a la deposición. Se fabricaron dos grupos de muestras, sobre las primeras se depositó TiN, en condiciones de $8,3 \times 10^{-3} \mathrm{mbar},-60 \mathrm{~V}, 100 \mathrm{sccm}$ (centímetros cúbicos estandar por minuto) y dos intensidades 40 y 100A, mientras que el otro grupo se realizó una deposición previa de $\mathrm{Al}$ durante 15 minutos a $40 \mathrm{~A}$, el voltaje de polarización, flujo de gas e intensidad aplicados fueron los mismos que para el TiN.

Los recubrimientos fueron analizados mediante Rayos X (XRD) con un difractómetro Bruker AXS D8 discover con detector de centelleo utilizando ángulo rasante de $1^{\circ}$ con radiación de $\mathrm{Cu}-\alpha$. Se trabajó con una amplitud de ángulo $2 \theta$ entre $20^{\circ}$ y $90^{\circ}$, con un paso de $0,03^{\circ}$. La capa superior del recubrimiento fue examinada en un rugosímetro Láser Confocal Sensofar PL $\mu 2300$ evaluando un área de 20x20mm.

Para la caracterización microestructural de las muestras se realizaron cortes perpendiculares a la capa del recubrimiento de un espesor de $5 \mathrm{~mm}$. Posteriormente se embutieron, desbastaron y pulieron con pasta de diamante de $3 \mu \mathrm{m}$. Los posibles residuos del pulido se limpiaron con alcohol. Se tomaron imágenes y espesores de diversa zonas de las secciones mediante un microscopio óptico Olympus GX51 y un microscopio electrónico de barrido Jeol Jfm 6500 f con 
filamento de emisión de campo tipo Schottkey, equipado con el sistema de microanálisis por dispersión de energía Oxford Inca y cámara de infrarrojos (EDX) para el análisis semicuantitativo.

\section{RESULTADOS Y DISCUSIÓN}

El TiN posee una estructura tipo $\mathrm{NaCl}$, con un parámetros de red de $\mathrm{a}=0,424 \mathrm{~nm}$, donde el nitrógeno ocupa los huecos octaédricos dentro de la red fcc.

Las Figuras 3 y 4 muestran los patrones de difracción de rayos $\mathrm{X}$ obtenidos en los recubrimientos de $\mathrm{TiN}$, con y sin la capa de aluminio, agrupados según variación de intensidad de corriente empleada en el procesado. La Figura 3 corresponde a las muestras desbastadas y la Figura 4 en estado pulido. En los patrones de difracción se han señalado mediante símbolos todos los compuestos aparecidos en el material base. Además, se han indicado los planos de los picos principales de crecimiento del TiN.

El patrón de difracción del TiN (CAS Number: 12033-66-8) presenta la mayor intensidad en el plano $(200)$ a un ángulo $2 \theta$ de $49,895^{\circ}$. En $2 \theta=42,842^{\circ}$ se encuentra el plano (1 11 ) con una intensidad relativa $\mathrm{I}_{(111)} / \mathrm{I}_{(200)}=0,72$. La tercera reflexión más relevante, con una intensidad relativa $\mathrm{I}_{(220)} / \mathrm{I}_{(200)}=0,45$ aparece en $2 \theta=73,233$.

Para evaluar la evolución de los planos de crecimiento de los resultados, se han comparado las intensidades relativas de los planos principales de los difractogramas de las muestras experimentales, y contrastado posteriormente con el patrón indicado anteriormente.

Se puede apreciar como en estado desbastado, Figura 3 a y b, tanto para intensidad de $40 \mathrm{~A}$ como para 100A, hay presencia de magnesio $\left(\mathrm{Mg}, \mathrm{MgO}, \mathrm{Mg}_{17} \mathrm{Al}_{12}\right)$. En estado pulido, Figura $4 \mathrm{a}$ y b, aparecen suprimidos todos los picos relevantes al magnesio. La ausencia de material base indica que el recubrimiento obtenido es más homogéneo y denso y, por tanto, la capa será más continua y sus cualidades protectoras mejorarán.

Para ambas preparaciones superficiales, Figura $3 a$ y b y Figura 4a y b, el aumento de intensidad de 40 a 100A provoca una disminución de la intensidad relativa del plano (2 2 0) y un aumento de la intensidad relativa del plano (1 11 1). El plano (200) no sufre a penas trasformación, como se podrá observar en la Tabla I (Relación de intensidades de difracción del TiN). Por lo tanto, el plano ( $\left.\begin{array}{llll}1 & 1 & 1\end{array}\right)$ se establece como el principal, su crecimiento favorece la orientación de los granos en este plano, generando una estructura tipo columnar. Esto es producido porque las islas crecen más rápido longitudinalmente según la dirección (1 11 1) quedando facetadas por los granos orientados en la (2 0 0), en la que crecen más lentamente (los átomos poseen mayor movilidad en estos planos), esto es lo que produce la estructura superficial que se podrán ver en las imágenes superficiales posteriores [16, 23].

Se podría decir que empleando una intensidad de $40 \mathrm{~A}$, el recubrimiento crece de forma aleatoria, ocupando diferentes direcciones cristalinas en función de la llegada de material al substrato, mientras que a $100 \mathrm{~A}$, se produce un aumento de energía de los iones, favoreciendo el crecimiento del plano (1 11). Esta tendencia fue estudiada por Li [xiv], Rauschenbach [24] y Mathieu [16], donde explicaban que el cambio de las condiciones energéticas produce una variación del espesor de la capa. El aumento del espesor genera un cambio en el tipo de crecimiento del recubrimiento, desde una organización aleatoria con granos de forma equiaxial a bajos espesores, a una morfología columnar con orientaciones preferentes planares cuando se obtienen espesores altos.

Las Figuras 3c y $4 \mathrm{c}$ reflejan los resultados obtenidos del recubrimiento de $\mathrm{TiN}$ con capa de $\mathrm{Al}$ intermedia en condiciones de intensidad de 40 A. En esta situación, el crecimiento preferencial del TiN no corresponde con el plano (1 1 1), sino que se da en el plano (2 20 ), plano de menor energía de parada. En nuestro caso, el cambio planar puede deberse a las tensiones derivadas de la incorporación de la capa intermedia de aluminio, que produce que el TiN crezca más tensionado. El crecimiento en el plano (2 2 0) progresa cuando la energía que llega al substrato o al recubrimiento es muy alta, incrementando las tensiones; su desarrollo se produce tras completarse el crecimiento en los planos de menor energía superficial (2 000 ) y de menor energía de deformación (1 111 ).

Los patrones de difracción mostrados en las Figuras 3c y $4 \mathrm{c}$ no detectan aluminio en los recubrimientos, presencia que si se verá más adelante en los resultados de la microscopía electrónica de barrido. Este hecho pudiera ser porque el espesor del TiN próximo de $1,8 \mu \mathrm{m}$ sea suficientemente alto

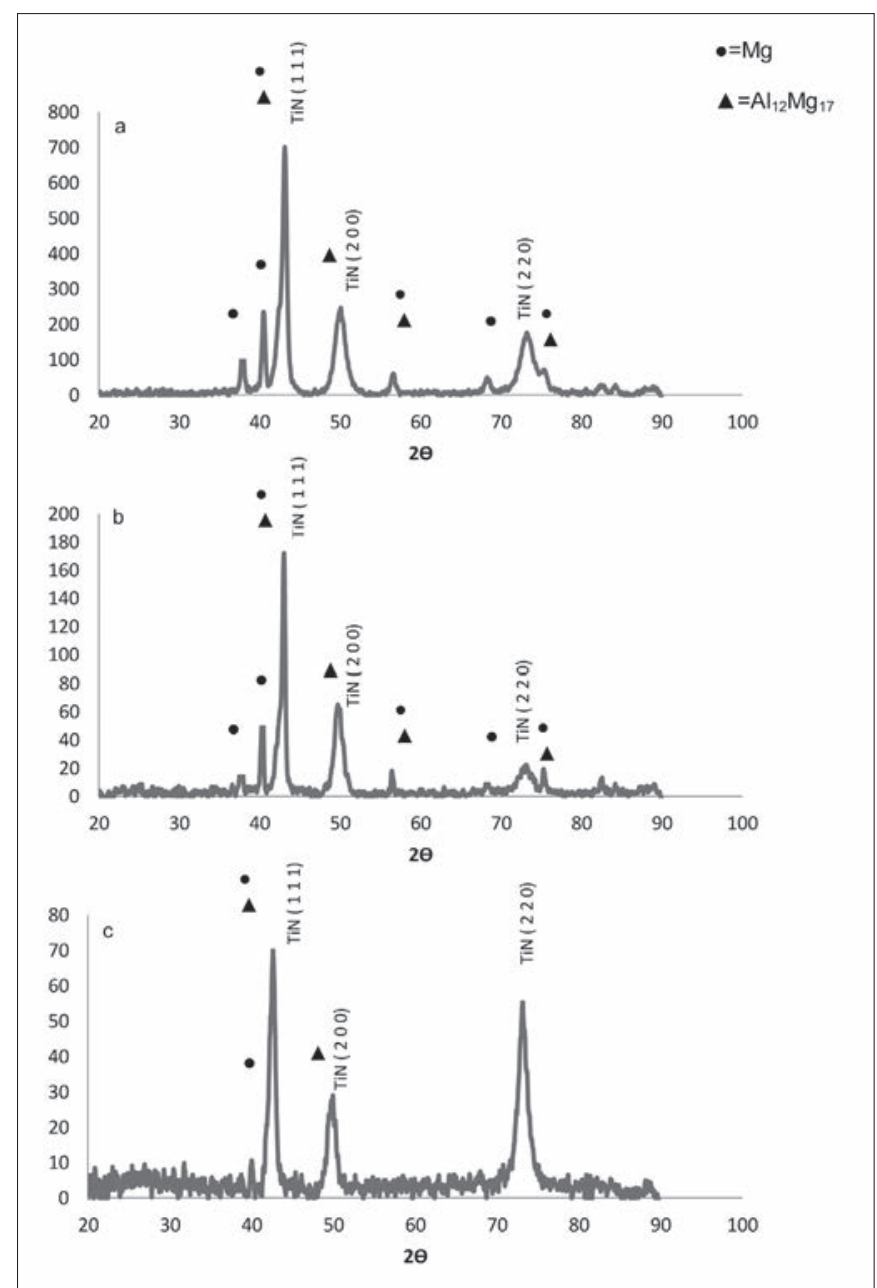

Figura 3 (a-c): Difractogramas de los recubrimientos depositados sobre Mg en estado desbastado. a) Recubrimiento de TiN a intensidad 40A, b) Recubrimiento de TiN a intensidad 100A c) Recubrimientos de $\mathrm{Al}+\mathrm{TiN}$ a intensidad $40 \mathrm{~A}$. 
como para que el haz, cuyo ángulo es de $1^{\circ}$, no llegase a penetrar hasta dicha capa, no revelándose su existencia. Se calculó mediante el programa Absorbdx diffracplus la pentetración del haz del TiN en todo el rango de barrido y se confirmó que resulta menor a $2 \mu \mathrm{m}$.

La Tabla I presenta el ratio de intensidades entre los planos $\left(\begin{array}{lll}2 & 0 & 0\end{array}\right) /\left(\begin{array}{lll}1 & 1 & 1\end{array}\right)$ y $\left(\begin{array}{lll}2 & 2 & 0\end{array}\right) /\left(\begin{array}{lll}1 & 1 & 1\end{array}\right)$ que son los picos de mayor intensidad cuya evolución vamos a estudiar. Los valores de la relación de estos planos obtenidos del patrón aparecen en la cabecera de la tabla, en las filas consecutivas se indican los obtenidos en nuestros resultados. Para conocer las intensidades relativas se han restado de los difractogramas los valores de los picos de magnesio que eran conocidos y se ha asumido que el resto de la intensidad recibida corresponde con el pico de TiN correspondiente. Se seleccionó como plano referencia el (1 111 ) por ser el de crecimiento preferente.

A continuación se evalúa la variación de la la rugosidad con los parámetros de fabricación. La Tabla II muestra los resultados de rugosidad obtenidos según los parámetros de fabricación descritos. La rugosidad fue medida mediante el parámetro Ra, valor promedio entre picos y valles del perfil. El valor de la rugosidad se halló por el promedio de 24 medidas adquiridas, 8 puntos de análisis en 3 líneas de estudio diferentes; los errores obtenidos promedio para las probetas en estado desbastado son

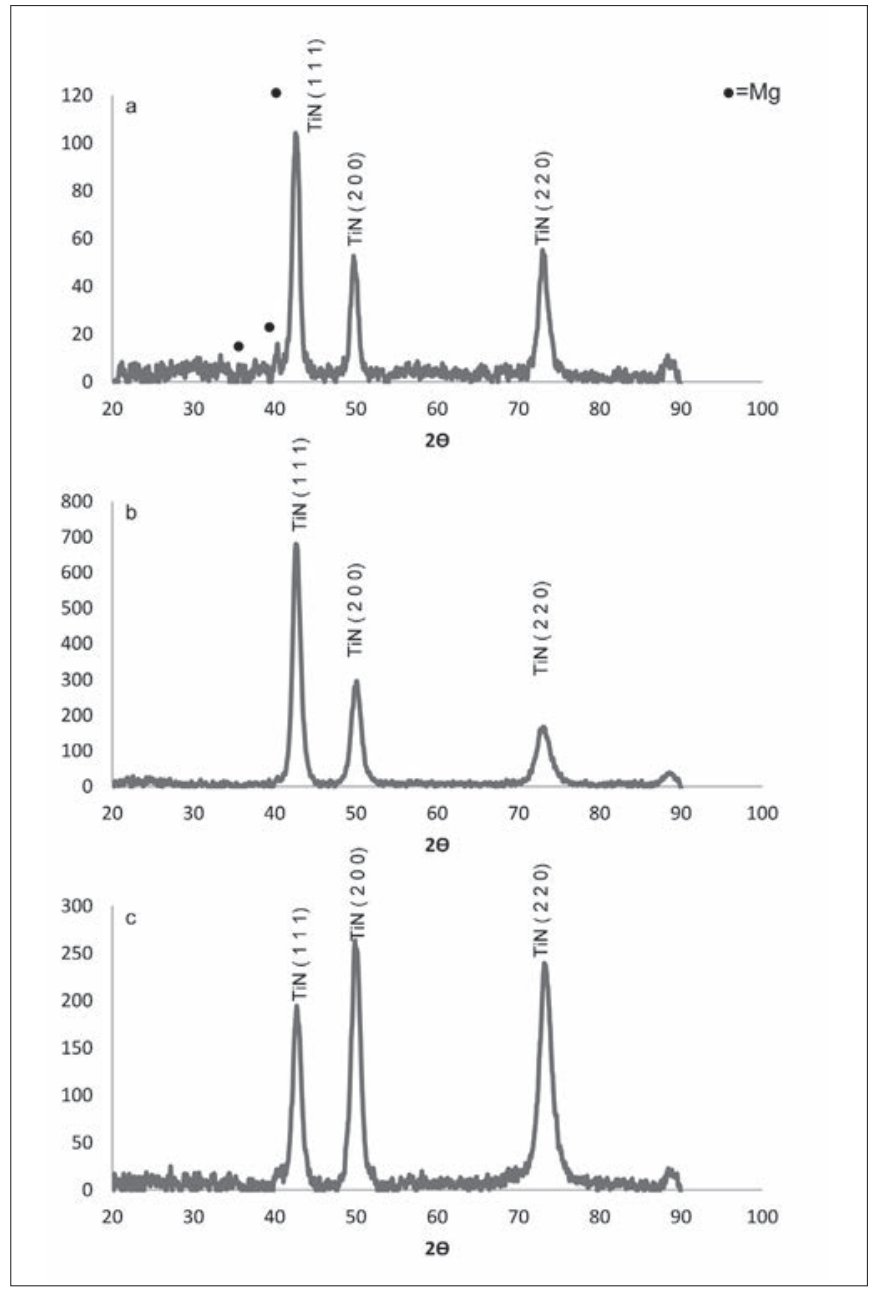

Figura 4 (a-c): Difractogramas de los recubrimientos depositados sobre Mg en estado pulido. a) Recubrimiento de TiN a intensidad 40A, b) Recubrimiento de $\mathrm{TiN}$ a intensidad $100 \mathrm{~A}$ c) Recubrimientos de $\mathrm{Al}+\mathrm{TiN}$ a intensidad $40 \mathrm{~A}$. de $0,07 \mu \mathrm{m}$ y en estado pulido de $0,03 \mu \mathrm{m}$. En estos resultados no se incluyen los relativos a la condición AM60 en 100A, debido a una desviación en la medición.

TABLA I: RELACIÓN DE INTENSIDADES DE DIFRACCIÓN DEL TIN.

\begin{tabular}{|c|c|c|c|}
\hline & & $\begin{array}{c}\text { Patrón } I_{(200)} / I_{(111)} \\
=1,38\end{array}$ & $\begin{array}{c}\text { Patrón } I_{(220)} / I_{(111)} \\
=0,62\end{array}$ \\
\hline \multirow{3}{*}{$\begin{array}{c}\text { Estado } \\
\text { desbastado }\end{array}$} & TiN- 40A & 0,74 & 0,43 \\
\hline & TiN- 100A & 0,73 & 0,18 \\
\hline & $\begin{array}{c}\mathrm{Al}+\mathrm{TiN}- \\
40 \mathrm{~A}\end{array}$ & 0,56 & 1,69 \\
\hline \multirow{3}{*}{$\begin{array}{l}\text { Estado } \\
\text { Pulido }\end{array}$} & TiN- 40A & 0,87 & 0,91 \\
\hline & TiN- $100 \mathrm{~A}$ & 0,66 & 0,31 \\
\hline & $\begin{array}{l}\mathrm{Al}+\mathrm{TiN}- \\
\quad 40 \mathrm{~A}\end{array}$ & 1,42 & 1,18 \\
\hline
\end{tabular}

Para este estudio de la rugosidad se incluyen además los resultados obtenidos para una aleación AZ91, aleación de magnesio con un $9 \%$ de aluminio y $1 \%$ zinc, fabricada en las mismas condiciones, a fin de poder establecer una tendencia condición-rugosidad. El resto de resultados de esta aleación no se incluyen en el artículo.

TABLA II: RUGOSIDAD DEL RECUBRIMIENTO TRAS EL PROCESADO.

\begin{tabular}{|c|c|c|c|c|}
\hline \multicolumn{2}{|c|}{$\begin{array}{c}\text { ESTADO } \\
\text { DESBASTADO }\end{array}$} & \multicolumn{2}{c|}{ ESTADO PULIDO } \\
\cline { 1 - 1 } AM60 & AZ91 & \multirow{2}{*}{ condición } & AM60 & $\begin{array}{c}\text { AZ91 } \\
\text { Ra }(\mu \mathrm{m})\end{array}$ \\
\cline { 1 - 2 } $\operatorname{Ra}(\mu \mathrm{m})$ & $\mathbf{R a}(\mu \mathrm{m})$ & & $\mathbf{R a}(\mu \mathrm{m})$ & 0,46 \\
\hline 0,44 & 0,59 & $\mathrm{TiN} / 40 \mathrm{~A}$ & 0,45 & 0,71 \\
\hline 0,57 & 0,73 & $\mathrm{TiN} / 100 \mathrm{~A}$ & - & 0,59 \\
\hline 0,64 & 0,66 & $\mathrm{Al}+\mathrm{TiN} / 40 \mathrm{~A}$ & 0,46 & 0,56 \\
\hline
\end{tabular}

Como se puede observar, la rugosidad menor aparece en condiciones de 40A. El incremento de la intensidad hasta 100A, o la introducción de una capa de aluminio intermedia, provocan un aumento de la rugosidad superficial final de las muestras como se comentó previamente.

Bajo las mismas condiciones de intensidad, las rugosidades tienen valores muy similares, independientemente de la preparación superficial intermedia. Tal y como explican Zieba et al. [25], pasado un tiempo de deposición largo la rugosidad superficial del recubrimiento es independiente del estado inicial del substrato.

Esta igualdad de los valores de rugosidad es debida a que, cuando tenemos superfies rugosas del substrato derivadas del estado de desbastado, la nucleación en los primeros estadios del TiN compensa las irregularidades. Las partículas/moléculas que llegan al substrato se depositan en las zonas discontinuas más profundas, zonas activadas energéticamente, reduciendo su energía con estas deposiciones. Una vez formados los núcleos estables comienza la formación de islas. Esta etapa se produce de forma similar en una superficie desbastada como pulida, igualando la rugosidad superficial inicial en los periodos de coalescencia y crecimiento, teniendo como resultado las superficies más lisas. En una superficie pulida, la nucleación de las islas genera una rugosidad inicial, acercándose a valores de una superficie desbastada, que se mantendrá en las siguientes etapas de crecimiento [25]. 
La Figura 5 muestra las micrografías de los diferentes recubrimientos. Se puede apreciar como independientemente del estado inicial del material base, con el aumento de la intensidad los recubrimientos obtenidos poseen mayor espesor, son más continuos y más adherentes, siguiendo en toda su longitud el perfil de la muestra. Estos datos corroboran lo presentado en los rayos $\mathrm{X}$, donde los picos del plano $\left(\begin{array}{lll}1 & 1 & 1\end{array}\right)$ del TiN, toma más relevancia, e incluso en estado pulido, los picos de magnesio desaparecen.

La variación de intensidad de 40 a $100 \mathrm{~A}$, produce un aumento en la cantidad del material evaporado del cátodo, aumentando la tasa de deposición del recubrimiento. Este incremento de la intensidad genera un aumento del voltaje del cátodo, afectando a la energía de los iones incidentes (que habían sido arrancados en condiciones más energéticas), depositándose con mayores velocidades. Este hecho deriva en un aumento de la adherencia entre la capa y el substrato, por la mejora de su unión mecánica. Cuando tenemos bajas intensidades, las tasas de deposición son más lentas, pudiendo tener un mayor control del recubrimiento pero, si la intensidad es demasiado baja, los iones incidentes no adquieren suficiente energía para adherirse en buenas condiciones al substrato, formalizando una mala unión.

No obstante, hay que tener en cuenta que si se produjese un aumento excesivo de la intensidad, y del consecuente aumento del voltaje, puede aparecer un crecimiento del efecto rebote de los iones incidentes, conocido como re-sputtering. Este re-sputtering provoca un incremento en la rugosidad de la superficie por fenómenos erosivos, puesto que aumenta la diferencia entre las partículas depositadas y aquellas que han sido arrancadas, adquiriendo una topografía más abrupta [26]. En este trabajo, este efecto no es detectado de manera constante, hay un aumento de la rugosidad pero no aparece un efecto erosivo, es decir, no hay una reducción aparente del espesor.

La introducción de la capa de aluminio, Figura 5 c y 5d, a una intensidad de 40A no mejora la unión del recubrimiento con el substrato; esta intensidad no resulta suficiente para formar una intercara estable. Por lo tanto la introducción
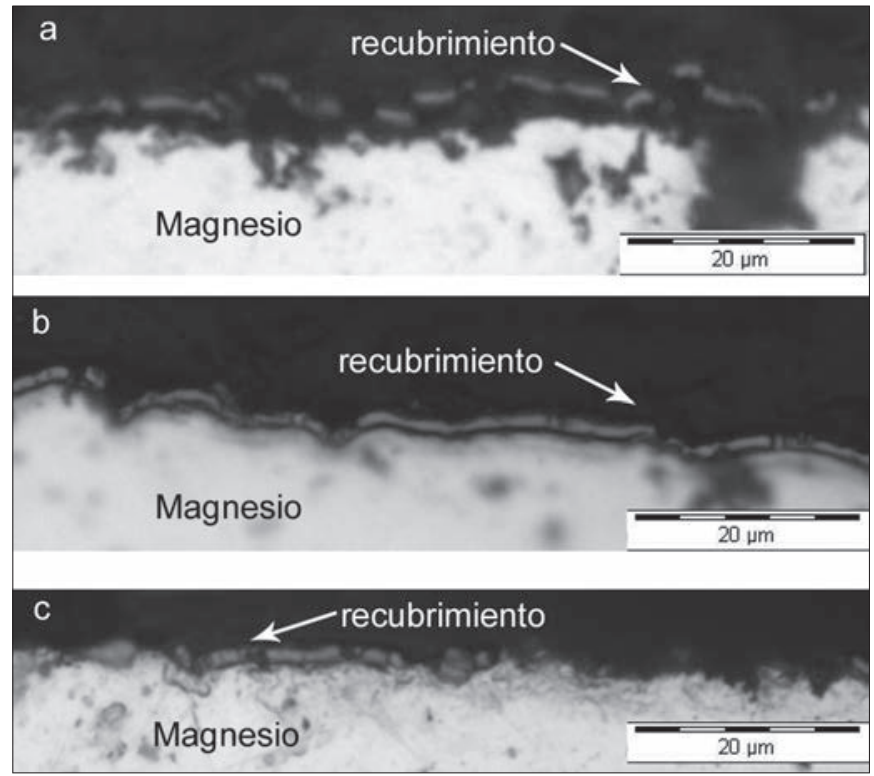

de aluminio en estas condiciones no mejora la adherencia con el substrato, pues el recubrimiento sigue apareciendo roto y quebradizo, dejando bastantes zonas del substrato al descubierto.

Las características detectadas en la inspección del corte transversal de las muestras quedan también de manifiesto en la inspección superficial de las capas, Figura 6.

La Figura 6a, b y c muestran que en estado desbastado el recubrimiento crece respetando las irregularidades del substrato originadas en el proceso de desbaste. En la condición de intensidad 40 A, Figura $6 a$, se ve una superficie muy heterogénea, distinguiéndose claramente líneas de desbastado y zonas desnudas del substrato, (hecho que también se vio en rayos $\mathrm{X}$ ).

A medida que las condiciones de procesado varían, aumentando de 40 a 100 A, los recubrimientos aparecen más homogéneos. Se puede observar como existe un cambio en la morfología de crecimiento de la capa, tal y como se detectó en los diagramas de difracción. Este hecho se aprecia mejor en estado pulido, Figuras $6 \mathrm{~d}-\mathrm{f}$, ya que posee menos líneas del rayado que enmascaran el crecimiento.

La Figura 6d muestra la superficie del recubrimiento de TiN en estado pulido, donde aparecen áreas de magnesio sin recubrir, corroborando los resultados de rayos $\mathrm{X}$. La Figura 6e permite apreciar un crecimiento en forma de islas perpendiculares al substrato (tal y como se explicó en la introducción en la Figura 2) diferenciadas por limites de grano y las zonas de depresión surgidas en la interferencia de unas islas con otras, véase Figura 1. Finalmente, en la imagen de la Figura $6 f$ se muestra la superficie de la muestra de TiN con capa de anclaje de aluminio, en ella se aprecia que la orientación ha cambiado hacia una superficie ausente de islas perpendiculares al substrato; cambio que quedó reflejado en las Figuras 3 y 4 donde la incorporación de la capa de Al hace que el plano preferente de crecimiento sea el $\left(\begin{array}{lll}2 & 0 & 0\end{array}\right)$.

Las micrografías de la Figura 6, muestran también la aparición de macrogotas superficiales. Estas macrogotas son gotas de titanio puro que se generan durante la deposición
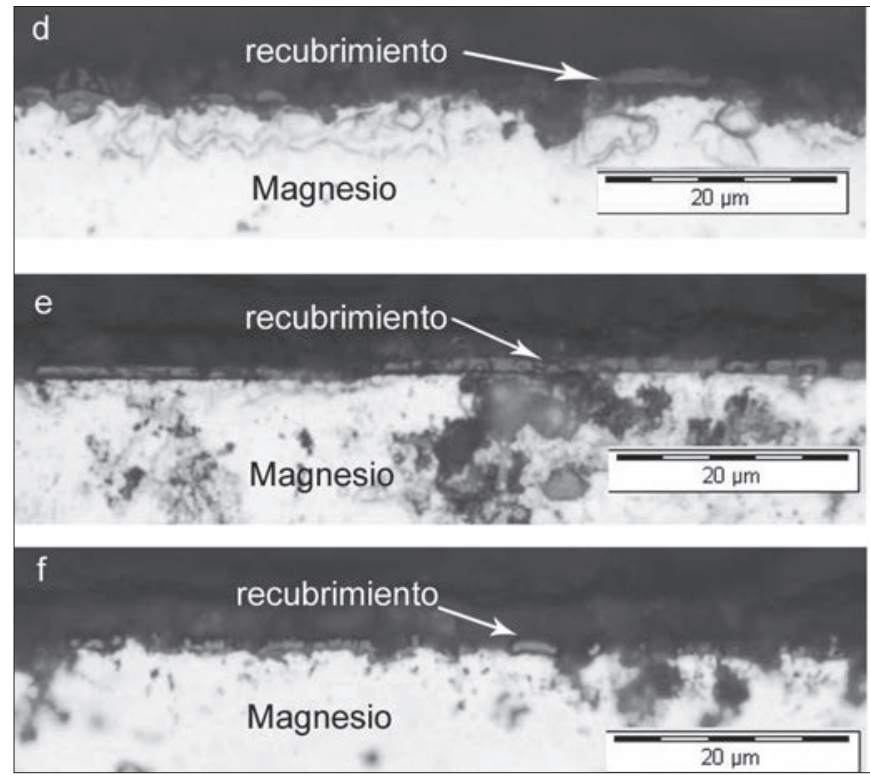

Figura 5: Micrografías transversales de la aleación AM60, estado desbastado y posteriormente recubierta con a) TiN a 40 A, b) TiN a 100 A, c) Al+TiN 40 A. Micrografías transversales AM60 estado pulido y posteriormente recubierta con d) TiN a 40 A, e) TiN a 100 A, f) Al+TiN 40 A. 

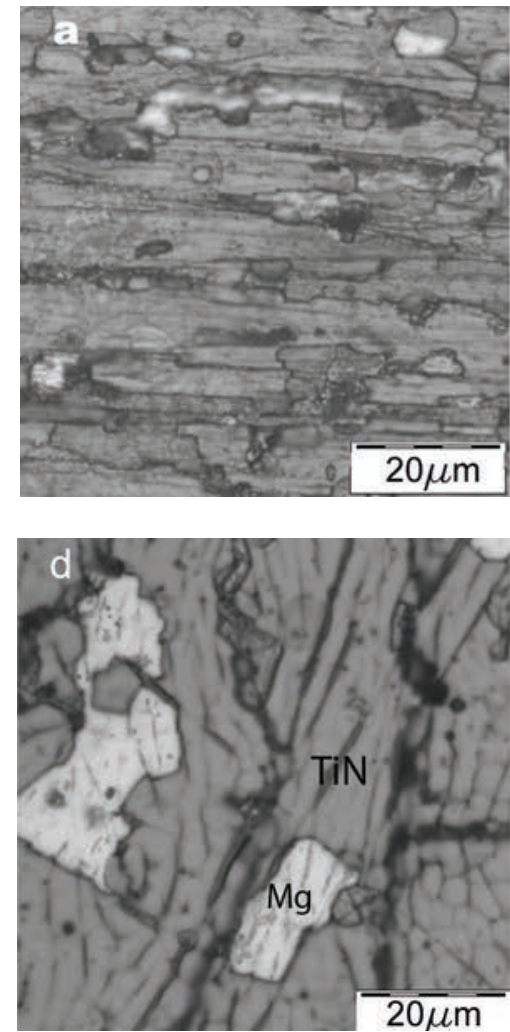
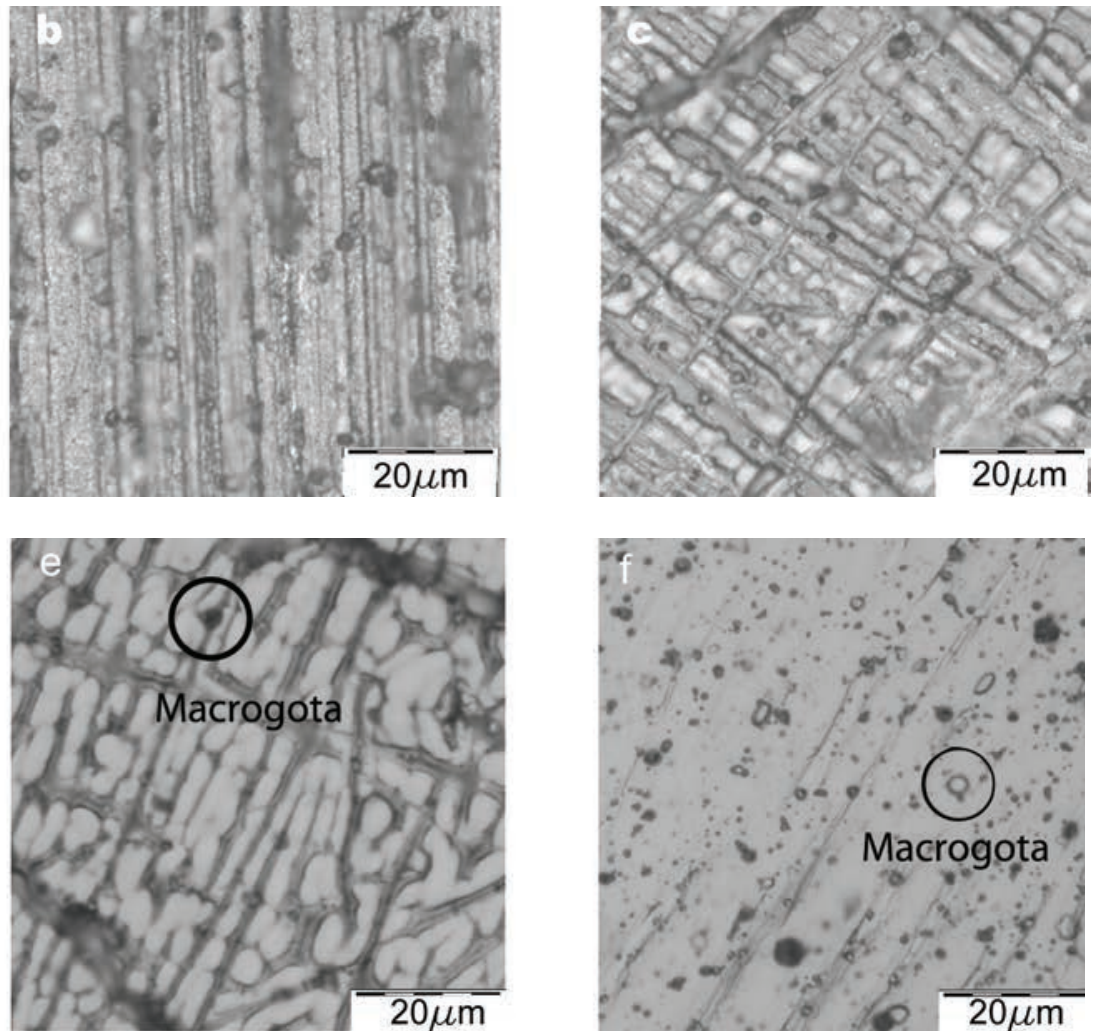

Figura 6: Imágenes superficiales de la aleación AM60, estado desbastado y posteriormente recubierta con a) TiN a 40 A, b) TiN a 100 A, c) Al+TiN 40 A. Imágenes superficiales AM60 estado pulido y posteriormente recubierta con d) TiN a 40 A, e) TiN a 100 A, f) Al+TiN 40 A.

del recubrimiento por la fusión parcial del cátodo, quedando depositadas en el recubrimiento durante su crecimiento. $\mathrm{Su}$ existencia posee efectos negativos en la calidad del recubrimiento, introduce tensiones en él afectando a la adherencia y aumentando la rugosidad. En estado pulido se observa mayor cantidad de éstas, es probable que sea porque la superficie al tener mejor acabado superficialmente permite detectar mejor su presencia. En estado desbastado las líneas de la preparación superficial enmascararán algunas de estas gotas, ya que realmente su emisión depende de las variables de fabricación y no del substrato.

Se realizó un estudio del tamaño y área ocupada de las macrogotas en estado pulido en función de los parámetros de fabricación, los resultados obtenidos están reflejados en la Tabla III. Los resultados en estado desbastado no se incluyeron pues las líneas de rayado no permitían diferenciar bien las macrogotas.

TABla III. ANÁLISIS DEL tAMAÑo Y DISTRIBUCión DE LAS MACROGOTAS EN RECUBRIMIENTOS EN ESTADO PULIDO.

\begin{tabular}{|l|c|c|c|}
\hline & $\begin{array}{c}\text { Tamaño } \\
\text { medio de } \\
\text { gota }(\mu \mathrm{m})\end{array}$ & $\begin{array}{c}\text { Área } \\
\text { superficial } \\
\text { gotas }\left(\mu \mathbf{m}^{2}\right)\end{array}$ & $\begin{array}{c}\text { Áreas gota/ } \\
\text { área estudio } \\
(\%)\end{array}$ \\
\hline $\begin{array}{l}\text { TiN pulido, } \\
\text { intensidad 40A }\end{array}$ & 0,133 & 0,349 & 0,615 \\
\hline $\begin{array}{l}\text { TiN pulido, } \\
\text { intensidad 100A }\end{array}$ & 0,213 & 0,803 & 1,415 \\
\hline $\begin{array}{l}\text { Al+TiN pulido, } \\
\text { intensidad 40A }\end{array}$ & 0,174 & 1,086 & 1,930 \\
\hline
\end{tabular}

El aumento de intensidad o la introducción de la capa de aluminio intermedia, produce un aumento en el tamaño y cantidad de macrogotas, incrementando la relación con el área superficial estudiada. Elevando el valor de intensidad a 100A, se produce mayor calentamiento local del cátodo, por lo tanto, mayor emisión de gotas de titanio que depositarán sobre la superficie del substrato o el recubrimiento creciente, dando lugar a las macrogotas.

Es probable que la condición en que la muestra posee una capa intermedia de aluminio procesada a una intensidad de $40 \mathrm{~A}$, permita detectar mejor las macrogotas, debido al cambio de crecimiento, véase Figura 1, que deja una superficie menos abrupta, sin granos facetados que puedan enmascarar su presencia, ver Figuras 6c y f. Además, en el esta condición, la introducción de la capa de aluminio previa favorece la emisión de estas macrogotas. Por un lado el cátodo de aluminio empleado para la deposición de la capa intermedia posee menor temperatura de fusión que el titanio, emitiendo mayor cantidad que él a 40 A. Por otro lado, la deposición de esta capa requiere de un tiempo de procesado de 15 minutos más que la capa sencilla de TiN, por lo que hay un mayor flujo total de macrogotas.

Finalmente, las imágenes obtenidas mediante SEM, Figura 7, muestran los resultados obtenidos a 100 A sobre la aleación AM60, en estado desbastado (Figura 7a) y pulido (Figura 7b).

La Figura $7 \mathrm{a}$ y $7 \mathrm{~b}$, muestra el recubrimiento de TiN que cubre la superficie del substrato, acoplándose a su geometría, con un espesor aproximado de 1,5 $\mu \mathrm{m}$. La Figura $7 \mathrm{a}$, donde el recubrimiento mostrado es sobre el substrato en estado desbastado, revela un recubrimiento poco continuo con 

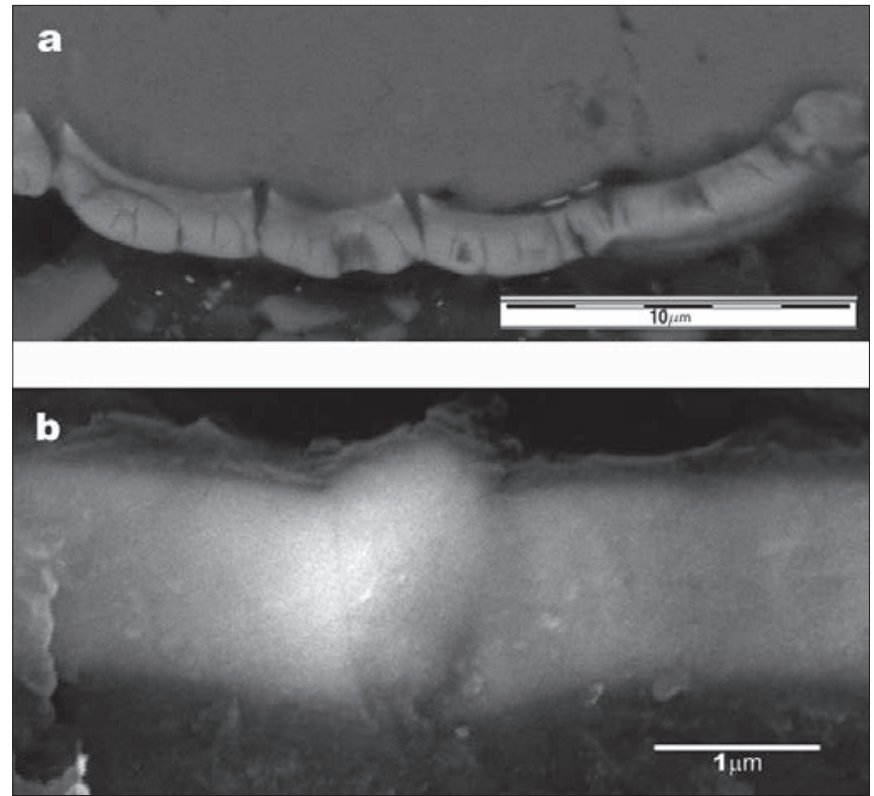

Figura 7. Imágenes del recubrimiento obtenidas mediante SEM depositadas a $100 \mathrm{~A}$ a) desbastado, b) pulido.

grietas perpendiculares a la superficie, aunque su deposición ha sido bastante homogénea cubriendo la geometría del substrato, es decir, ha reflejado las irregularidades propias de las piezas del magnesio. La ruptura del recubrimiento está originada por las tensiones generadas por el efecto de los iones bombardeantes que, al alcanzar el substrato encuentran mayor superficie sobre la que impactar. En cada uno de estos impacto se introducen pequeñas tensiones que deben ser adsorbidas por el substrato, la intercara y el recubrimiento, si estos no pueden reacomodarlas, se producirá el fallo del mismo.

La Figura $7 \mathrm{~b}$ muestra un recubrimiento denso y homogéneo, derivado de una mejor adhesión con el substrato y menor rugosidad, debido a la preparación en estado pulido. Además, se puede ver la estructura de una macrogota originada por la fusión del cátodo, que ha sido depositada sobre el magnesio, su diámetro es aproximadamente de $1 \mu \mathrm{m}$ y permite diferenciar las grietas que se forman en su perímetro como consecuencia de tensiones introducidas [27, 28].

\section{CONCLUSIONES}

Los resultados presentados muestran que, el aumento de la intensidad, produce un aumento en la tasa de evaporación de los iones incidentes, incrementando el espesor y calidad del recubrimiento. El crecimiento del recubrimiento de TiN se establece en el plano (1 111 ) que toma mayor relevancia con el aumento de la intensidad, sobre los otros planos $\left(\begin{array}{lll}2 & 0 & 0\end{array}\right)$ y $(22$ $0)$; este crecimiento se sucede en modo de columnas, dentro de la Zona T de crecimiento.

La rugosidad del recubrimiento no se ve afectada tras periodos largos de deposición; así como la preparación superficial inicial de las muestras no afecta notablemente a los valores finales obtenidos tras la deposición de TiN. Las micrografías obtenidas mediante microscopía óptica y de barrido mostraban como una superficie pulida del substrato puede favorecer la adherencia y homogeneidad de la capa, sin influir en exceso sobre su rugosidad. Se puede concluir que la rugosidad del substrato vendrá determinada por el crecimiento en las primeras etapas y los planos preferenciales de crecimiento, la aplicación de un pulido previo de la muestras origina recubrimientos más continuos y menos tensionados, favoreciendo la adherencia del substrato.

La introducción de una capa intermedia de aluminio en estas condiciones no beneficia la calidad del film, dejando una superficie más rugosa, con un crecimiento en el plano (2 2 0), plano de menor energía de parada, y una estructura tensionada, no favoreciendo la adherencia del mismo.

Tras los resultados obtenidos, se considera que la condición más óptima para la deposición de TiN sobre la aleación AM60 es la correspondiente a una intensidad de 100A, debido a la homogeneidad, continuidad y espesor de la capa.

\section{AGRADECIMIENTOS}

Los autores quieren agradecer al Ministerio de Ciencia e Innovación el apoyo financiero para el desarrollo del proyecto CENIT-MAGNO, donde se engloba esta investigación, así como a las empresas Antolín S.A. y TTC por su ayuda. Este trabajo fue subvencionado por la Comunidad de Madrid mediante un contrato de personal de apoyo a la investigación.

\section{BIBLIOGRAFÍA}

1. B.L. Mordike, T. Ebert. Eng. Magnesium. Properties-applications-potencial. Mat. Sci. A; 302: 37-45, 2001.

2. E. Aghion, B. Bronfin, D. Eliezer. The role of magnesium industry in protecting the envioroment . J. Mater. Process. Tech.; 117: 381-385, 2001.

3. A. Hikmet, S. Sandri. The effect of PVD coatings on the wear behaviour of magnesium alloys. Mater. Charact.; 58: 917-921, 2007.

4. M.A. Auger, Ao. Sánchez, J.M. Albella. Recubrimientos protectors de TiN y AlN: comportamiento frente a temperatura. Bol. Soc. Esp. Ceram.V.; 45,2; 75-79, 2006.

5. S. Kodambaka, S.V. Khare, V. Petrova, A. Viailions, I. Petrov, J.E. Greene. Determination of absolute orientation-dependent TiN $\left(\begin{array}{llll}0 & 0 & 1\end{array}\right)$ and TiN (1 1 1) step energies. Vacuum.; 74: 345-351, 2004.

6. J.E. Gray, B. Luan. Protective coatings on magnesium and its alloys -a critical review-. J. Alloy. Compd.; 336: 88-113, 2002.

7. H Ljungcrantsz, L. Hutman and J-E. Sundgren. Microestructural investigation of droplets in arc-evaporated TiN films. Surf. And Coat. Tech.; 63: 123-128, 1994

8. C.V. Thompson. Structure evolution during processing of polycrystalline films. Annu. Rev. Mater. Sci.; 30: 159-190, 2000

9. L.A. Dobrzanski, M. Polok, P. Panjan, S. Bugliosi, M. Adamiak Improvement of wear resistance of hot steels by PVD coatings deposition. J. Mater. Process. Tech.; 155-156: 1995-2001, 2004.

10. G. Abadias. Stress and preferred orientation in nitride-based PVD coatings. Surf. And Coat. Techn.; 202: 2223-2235, 2008.

11. M. Latteman, U. Helmersson, J.E. Greene. Fully dense, non-faceted 111textured high power impulse magnetron sputtering TiN films grown in the absence of substrate heating bias. Thin Solid Films; 518: 5978-5980, 2010.

12. C.V, Thomson. Structure evolution during processing of polycrystalline films. Annu. Rev. Mater. Sci.; 30: 159-190, 2000

13. J.M. Albella, I. Jimenez, C. Gómez-Aleixandre, A. Alberdi. Materials and vapour-phase techniques for the síntesis of ceramic coating. Bol. Soc. Esp. Ceram. V. 46,4;171-176, 2007.

14. T.Q. Li, S. Noda, Y. Tsujji, T. Ohsawa, H. Komiyama. Initial growth and texture formation during reactive magnetrón sputtering of TiN on Si (1 1 1). Dpto. Chemical System Engineering, University of Tokyo.

15. I. Petrov, P.B. Barna, L. Hultman, J.E. Greene. Microstructural evolution during film growth. J. Vac. Sci. Technol. A; 21, 5: 2003.

16. S. Mathieu, P. Ghekiere, D. Depla, R. De Gryse. Biaxial alignement in sputter deposited TiN films. Thin solid films 515, 1229-1249, 2006.

17. M.A. Auger, Ao. Sánchez, J.M. Albella. Depósito de TiN sobre herramientas de corte para uso industrial. Bol. Soc. Esp. Ceram. V.; 43,2; 488-490, 2004

18. S. Zhang, W. Zhu. TiN coatingof tool steels: a review. J. Mater. Process. Techn, 39: 165-177, 1993. 
19. K. Tuffy, G. Byrne, D. Dowling. Determination of the optimum TiN coating thickness on WC inserts for machining carbo steels J. Mater. Process. Tech.; 155-156: 1861-1866, 2004.

20. S. Mathieu, D. Depla, R. De Gryse. Journal of Physics: Conference Series; 100,2008

21. A. Mumbarak, E.B. Hamzah. Deposition and chaacterization of TiN-coated steels at various $\mathrm{N} 2 \mathrm{gAs}$ flow rates with constant etching by using CA-PVD technique. Surf. Rev. and Lett. 14,1; 93-100, 2007.

22. K. Nakamura, A. Wajayama, K. Yukimura. Effects of reactive gas addition on ionization of metal atoms in dropler-free metal ion surce. Surf. Coat. Tech.; 201, 6655-6659, 2007.

23. J.A. Thornton. High Rate thick film growth. Annu. Rev. Mater. Sci.; 7: 239260, 1977.

24. B. Rauschenbach, J.W. Gerlach. Texture development in titanium nitride films grown by low-energy ion assisted deposition. Crys. Res. Technol.; 35, 6-7: 675-688, 2000
25. L. Zieba, J. Jaglarz, M. Dabrowski, R. Duraj, J. Cisowski, J. Jurusik. Surface topography investigations of TiN layers on different substrates. Rev. Adv. Mater. Sci.; 15: 63-68, 2007

26. M. Pichel, G. Conejero, R. Barea, D. Val, M. Carsí, N. Candela, Efecto del voltaje y el flujo de gas en la rugosidad de recubrimientos de TiN fabricados por AC-PVD sobre aleaciones de magnesio. XII Congreso Nacional de Tratamietos Térmicos y de Superficie. 391-400, 2010.

27. A. Mumbarak, P. Akhter, M.R.M. Toff, E. Hamzah, I.A. Qazi. Macrodoplets reduction and growth mechanism in cathodic arc physical vapour deposition of TiN Films. Surf. Rev.. Lett.; 15, 5: 653-659, 2008.

28. K. Nakamura, A. Wajayama, K. Yukimura. Effects of reactive gas addition on ionization of metal atoms in dropler-free metal ion surce. Surf. Coat. Tech.; 201: 6655-6659, 2007.

Recibido: 29/08/2012

Recibida versión corregida: 12/03/2013

Aceptado: 19/03/2013 